\title{
Biomimetic Soft Polymer Microstructures and Piezoresistive Graphene MEMS Sensors Using Sacrificial Metal 3D Printing
}

\author{
Amar M. Kamat,* Yutao Pei, Bayu Jayawardhana, and Ajay Giri Prakash Kottapalli
}

Cite This: ACS Appl. Mater. Interfaces 2021, 13, 1094-1104

Read Online

ABSTRACT: Recent advances in 3D printing technology have enabled unprecedented design freedom across an ever-expanding portfolio of materials. However, direct $3 \mathrm{D}$ printing of soft polymeric materials such as polydimethylsiloxane (PDMS) is challenging, especially for structural complexities such as high-aspect ratio $(>20)$ structures, 3D microfluidic channels $(\sim 150 \mu \mathrm{m}$ diameter), and biomimetic microstructures. This work presents a novel processing method entailing 3D printing of a thin-walled sacrificial metallic mold, soft polymer casting, and acidic etching of the mold. The proposed workflow enables the facile fabrication of various complex, bioinspired PDMS structures (e.g., 3D double helical microfluidic channels embedded inside high-aspect ratio pillars) that are difficult or impossible to fabricate using currently available techniques. The microfluidic channels are further infused with conductive graphene nanoplatelet ink to realize two flexible piezoresistive microelectromechanical (MEMS) sensors (a bioinspired flow/tactile sensor and a dome-like force sensor) with embedded sensing elements. The MEMS force sensor is integrated into a Philips 9000 series electric shaver to demonstrate its application in "smart" consumer products in the future. Aided by current trends in industrialization and miniaturization in metal 3D printing, the proposed workflow shows promise as a low-temperature, scalable, and cleanroom-free technique of fabricating complex, soft polymeric, biomimetic structures, and embedded MEMS sensors.

KEYWORDS: 3D printing, additive manufacturing, MEMS, flow sensor, pressure sensor, embedded sensing, graphene, piezoresistivity, bioinspiration, microfluidics

\section{INTRODUCTION}

Three-dimensional (3D) printing, also known as additive manufacturing $(\mathrm{AM})$, is a disruptive technology that enables the fabrication of complex-shaped structures using a tool-less processing workflow. 3D printing or AM (which can be further classified into seven categories, viz., binder jetting, directed energy deposition, material extrusion, material jetting, powder bed fusion (PBF), sheet lamination, and vat photopolymerization $^{1}$ ) can be broadly defined as a technique to fabricate objects directly from a 3D computer-aided design (CAD) model, thereby enabling unprecedented design freedom. Although the $3 \mathrm{D}$ printing process typically builds up objects in a layer-by-layer fashion, recent work ${ }^{2}$ has also demonstrated the ability to realize volumetric $3 \mathrm{D}$ printing using tomographic reconstruction. The material portfolio of $3 \mathrm{D}$ printing has expanded greatly to include a wide variety of metals (e.g., stainless steel, titanium, aluminum, nickel-based alloys), ${ }^{3}$ ceramics (e.g., silicon carbide, alumina, titanium dioxide, lead zirconate titanate or "PZT", hydroxyapatite), ${ }^{4}$ and polymers (acrylates, epoxides, polyamide, polylactic acid, or "PLA", acrylonitrile butadiene styrene or "ABS", polycarbonate). ${ }^{5}$ In spite of the ever increasing materials repertoire, it is still challenging to $3 \mathrm{D}$ print several important soft materials ${ }^{6}$ such as polydimethylsiloxane (PDMS), hydrogels, and liquid crystals.
PDMS is a transparent, biocompatible, gas-permeable, and moldable (submicron resolution) elastomer that finds applications in microfluidics, ${ }^{7}$ organ-on-a-chip, ${ }^{8}$ membranes, ${ }^{9}$ tissue engineering, ${ }^{10}$ soft robotics, ${ }^{11}$ and flexible electronics, ${ }^{12}$ among others. Traditionally, PDMS structures such as microfluidic devices have been fabricated using soft lithography. ${ }^{13}$ Although this fabrication technique is mature and allows fine feature sizes (e.g., $5 \mu \mathrm{m}$ microfluidic channel width), it is labor-intensive and is beset by problems such as restrictions in fabricating 3D structures monolithically, low throughput, and an inherent incompatibility with automated manufacturing, ${ }^{14,15}$ prompting researchers to explore alternatives. Song et al. ${ }^{16}$ and Singh et al. ${ }^{17}$ cast PDMS around a metal wire (solder and copper, respectively) coiled in various $3 \mathrm{D}$ shapes (e.g., circular, helical, etc.) and subsequently removed the wire either manually or by heating it beyond its melting point, thereby creating a 3D microfluidic channel inside the PDMS block. Other researchers $^{18,19}$ utilized a similar sacrificial strategy by manually

Received: November 30, 2020

Accepted: December 23, 2020

Published: January 4, 2021 
embedding small pieces of hydrogel in the shape of the microchannels followed by PDMS casting and hot water washing to realize the microchannels. Owing to rapid advances in $3 \mathrm{D}$ printing technology around 2015 , subsequent studies used $3 \mathrm{D}$-printed sacrificial scaffolds using materials such as sugar, ${ }^{20}$ isomalt, ${ }^{21} \mathrm{ABS},{ }^{22}$ wax, ${ }^{23}$ acrylic, ${ }^{24}$ polyvinyl alcohol (PVA), ${ }^{25,26}$ and so on that were later either dissolved in a suitable solvent or removed manually ${ }^{24}$ from the cured PDMS block. Femmer et $a l^{27}$ extended the sacrificial molding strategy beyond microfluidics applications to fabricate 3D membrane architectures in PDMS.

Direct 3D printing of complex PDMS structures is not a trivial task owing to its low elastic modulus $(\sim 0.75-3 \mathrm{MPa})$ and long curing times $(\sim 10-30 \mathrm{~min})$ and has thus been the focus of recent research in material development. The research group of Lewis ${ }^{6}$ pioneered the direct ink writing (DIW) technique for viscoelastic materials, which relies upon customized thixotropic inks (including $\mathrm{PDMS}^{28}$ ) that behave as fluids when the extrusion pressure exceeds their yield stress and are deposited as solids once they are extruded from the nozzle. Roh et al. ${ }^{29}$ used a multiphase ink comprising cured PDMS microbeads, uncured PDMS liquid precursor, and water to $3 \mathrm{D}$ print several structures using the DIW process; however, due to the nature of the ink, the structures were necessarily porous. Femmer et al. ${ }^{30}$ formulated a PDMS photoresist that could be photo-crosslinked using digital light processing in a layer-by-layer fashion to fabricate 3D membrane architectures similar to those reported by the same authors elsewhere. ${ }^{27}$ Hinton et al. ${ }^{31}$ printed complex 3D structures such as helical tubes and perfusable manifolds using the so-called freeform reversible embedding method; they used a commercially available material extrusion printer to print the widely used Sylgard 184 PDMS resin inside a hydrophilic support bath, following which the PDMS was cured and the support bath was washed away in a phosphate buffered saline solution to release the 3D PDMS structure. More recently, Ozbolat et al. ${ }^{32}$ evaluated the 3D-printability of three different concentrations of PDMS solutions to print a human nose model using the material extrusion process and reported superior mechanical properties and cell adhesion of the 3D-printed samples compared to their casted counterparts due to lower porosity and higher surface roughness, respectively.

Although considerable progress has been evidently made in the $3 \mathrm{D}$ printing of soft materials such as PDMS, some limitations persist. The low elastic modulus of PDMS (and indeed most polymers) makes it difficult to directly 3D print self-supporting structures such as, for instance, high-aspect ratio (HAR) $(>20)$ micropillars. HAR micropillars find a multitude of applications such as bioinspired dry adhesion, ${ }^{33}$ bioinspired cilia structures for microfluidic manipulation ${ }^{34}$ and underwater flow sensing, ${ }^{35-37}$ shear stress sensing in turbulent flow, ${ }^{38}$ measurements in cell and protein mechanics, ${ }^{39}$ microneedles for transdermal drug delivery, ${ }^{40}$ and so on. However, fabricating HAR micropillars in PDMS using soft lithography is difficult owing to its low tensile strength $(\sim 5 \mathrm{MPa})$ that often causes micropillar failure during the demolding process. Although novel techniques such as direct-drawing from PDMS microdroplets $^{41,42}$ and PDMS double casting with plasma surface modification $^{43}$ to fabricate HAR micropillars have been demonstrated, these methods do not offer the same design freedom as $3 \mathrm{D}$ printing. In addition to the inability to fabricate HAR structures, it is generally difficult to render fine features $(\sim 150 \mu \mathrm{m})$ in $3 \mathrm{D}$-printed PDMS structures ${ }^{32}$ due to the long curing time and high viscosity of PDMS.
In this work, we introduce a novel processing flow to fabricate complex 3D structures made of soft polymers. Our approach utilizes a 3D-printed, metallic, thin-shelled, sacrificial mold into which the soft polymer is cast and cured. Following this, the metallic mold is etched in an acidic solvent that dissolves the thin-shelled mold without affecting the polymer, thus releasing the desired polymeric structure. The proposed workflow can be used for the facile fabrication of various structural complexities (e.g., HAR micropillar arrays, membranes, 3D microfluidic channels networks, lattices, or a combination of one or more of the above) that are difficult to fabricate using conventional methods. We demonstrated the versatility of our approach by fabricating various complex PDMS microstructures such as double helical microfluidic channels inside a HAR pillar, serpentine microfluidic channels inside a HAR cantilever, a 2D array of micropillars of varying heights inspired by the biological "stereocilia" structure, and HAR micropillars (AR = 50). Further, internal microfluidic channels present interesting possibilities for the design and fabrication of flexible microelectromechanical (MEMS) sensors with embedded 3D sensing elements ${ }^{44,45}$ and integrated circuitry. ${ }^{46}$ We explored these possibilities by using our processing workflow to fabricate two different kinds of piezoresistive MEMS sensors: a hair-like tactile and flow sensor comprising a HAR PDMS pillar with grapheneinfused internal microchannels inspired by the mechanoreception principles of arthropod filiform hairs ${ }^{47}$ and fish neuromasts ${ }^{48}$ and a force sensor comprising a PDMS hemisphere with double helical graphene-infused microchannels. Graphene was chosen as the piezoresistive sensing element in the PDMS microfluidic channels due to its high gauge factor and reliable piezoresistive behavior. ${ }^{49}$ The MEMS dome sensor was further integrated into a consumer product, namely, the Philips 9000 series personal shaver to demonstrate its feasibility in sensing shaving forces, with a view to potentially using the sensor data to take corrective action and reduce skin irritation. ${ }^{50}$

Traditionally, MEMS sensors have been fabricated using conventional surface and bulk micromachining processes in the cleanroom, a combination of which is used to fabricate essential components such as the sensor structure, the sensing elements (e.g., strain gauges), and the associated complementary metaloxide-semiconductor electronics. Although cleanroom processes are capable of fabricating simple 3D MEMS structures, they are cumbersome, expensive, and heavily limited by the choice of materials (mainly silicon and SU-8) and achievable aspect ratios (ARs) of the structures. Moreover, the sensing elements, for example, piezoresistive strain gauges, are limited to planar (2D) designs, thus severely restricting the design space for MEMS sensors. Our sensor fabrication approach circumvents the above problems by allowing both the sensor structure and the sensing elements to possess intricate $3 \mathrm{D}$ shapes such as HAR structures and 3D microfluidic channels, thus significantly enhancing the design freedom. The proposed method thus establishes a cleanroom-free, repeatable, and low-temperature fabrication technique to develop complex soft polymer MEMS structures with embedded 3D sensing elements. The resulting flow and force sensors showed excellent sensitivity to controlled stimuli, thus validating the proposed processing workflow as a feasible technique for the facile fabrication of flexible MEMS sensors.

\section{RESULTS AND DISCUSSION}

Fabrication Process Workflow. The processing workflow consisted of four main steps as described below for an exemplar 


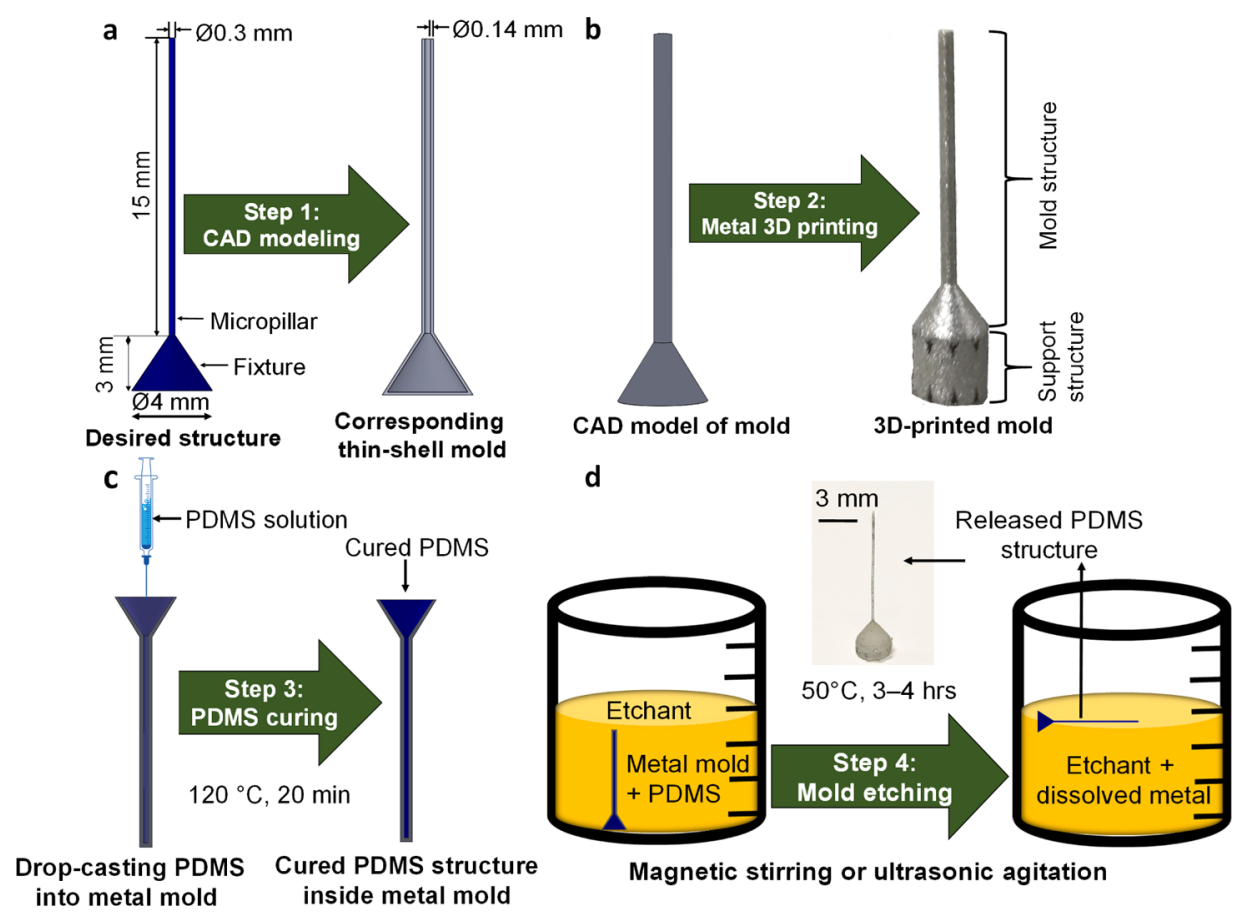

Figure 1. Proposed processing workflow. (a) CAD model of the thin-walled sacrificial metallic mold designed as the "negative" of the desired PDMS structure (in this case, a slender pillar with $\mathrm{AR}=50$ ). (b) 3D printing of the metallic mold using LPBF. (c) Drop-casting and curing PDMS inside the metallic mold. (d) Etching the sacrificial mold in an acidic solvent to release the desired PDMS structure.

case of fabricating a HAR micropillar with dimension of $300 \mu \mathrm{m}$ diameter, $15 \mathrm{~mm}$ length, and an AR of 50, a structure that usually presents great difficulties for fabrication using currently available techniques (3D printing or otherwise) for soft polymers.

Step l: Preparation of a CAD model (Figure 1a). A CAD of the sacrificial metallic mold was created as a thin-shelled cavity that was essentially a "negative" of the polymer structure to be fabricated. The shell had a wall thickness of $140 \mu \mathrm{m}$, which was the minimum permissible with the available metal 3D printer. The build direction was parallel to the axis of the HAR pillar to minimize support structures (required only at the bottom to connect the fixture to the build plate) and to ensure a good printing resolution in the build plane (i.e., to be able to print the $300 \mu \mathrm{m}$ hole in the mold in this particular case). The mold wall was thin enough to be etched within reasonable times $(\sim 3-4 \mathrm{~h}$ at $50{ }^{\circ} \mathrm{C}$ ) while thick enough to maintain the robustness of the structure during the $3 \mathrm{D}$-printing process, the latter being especially important while 3D-printing HAR structures.

Step II: Metal 3D Printing (Figure 1b). The CAD model of the sacrificial mold from Step I was 3D-printed in stainless steel using the laser powder bed fusion (LPBF) process. Briefly, this metal 3D printing process entails selectively melting and fusing the metallic powder feedstock using a focused and rapidly moving laser beam, after which the new powder layer is deposited and the process is repeated. In the LPBF process, the powder size distribution and the laser beam spot size dictate the resolution that in turn determines the lower limit of feature sizes in the resulting polymeric structure (or sensor). In this work, the laser spot size was $70 \mu \mathrm{m}$ and the stainless steel powder size distribution was in the range of $10-45 \mu \mathrm{m}$, leading to a minimum wall thickness of $\sim 140 \mu \mathrm{m}$ and a minimum hole diameter of $\sim 200 \mu \mathrm{m}$. It must be noted that although LPBF was used in this study, other techniques such as binder jetting can also be used to 3D-print the metallic mold. Further, any other 3D-printable metal or alloy ( copper, iron, etc.) can be used as the raw material as long as a solvent exists to etch it within reasonable times without affecting the polymer to be cast later. It is especially economical to use cheaper metal powders such as water-atomized pure iron as the molds are sacrificial.

Step III: PDMS Casting (Figure 1c). Commercially available PDMS was used as the soft polymeric material to be cast into the 3D-printed molds. Liquid PDMS solution was injected into the mold cavity and subsequently cured to form the solid PDMS microstructure inside the mold. Care was taken while injecting PDMS into the mold to ensure that the PDMS did not coat the outer surface of the mold as this would prevent the metal from getting etched in step IV.

Step IV: Mold Etching (Figure 1d). Finally, the thin-shelled mold containing cured PDMS was etched in an acidic solvent (5-10 wt $\% \mathrm{FeCl}_{3}, 20-40$ wt \% $\mathrm{HCl}$, balance $\mathrm{H}_{2} \mathrm{O}$ ) at $50{ }^{\circ} \mathrm{C}$ under ultrasonic agitation for a period of 3-4 h. The above solvent was chosen as it etches stainless steel, ${ }^{51}$ is compatible with PDMS, ${ }^{52}$ and does not cause swelling or mechanical degradation of PDMS (more details in the Supporting Information, Figures S1 and S2 and Table S1). Once the thinshelled mold was completely etched away, the released PDMS structure floated atop the etchant, after which it was cleaned ultrasonically in water for another $15 \mathrm{~min}$.

The processing methodology described in steps I-IV above can, in principle, also be used to fabricate multimaterial polymeric microstructures with different properties (density, Young's modulus, etc.) in different locations, an example of which is shown in the Supporting Information (Figure S3). Some salient features of our method compared to the sacrificial or direct $3 \mathrm{D}$ printing strategies employed in the literature are as follows:

i. As metals are much stronger than other materials used for sacrificial molds before (e.g., wax, ${ }^{23}$ isomalt, $^{21}$ sugar, $^{20}$ $\mathrm{ABS}^{22}$ and $\mathrm{PVA}^{25,26}$ ), very HAR mold structures (up to 
at least 100) can be printed easily, thus enabling the fabrication of HAR structures.

ii. The superior strength of metals also means that the sacrificial mold/scaffold can be thin-shelled (sometimes as thin as $140 \mu \mathrm{m}$ where the shell thickness is dictated by the minimum permissible feature size of the metal 3D printing process). This not only saves material but also ensures quicker etching later.

iii. Metal 3D printing processes, especially PBF and binder jetting, exhibit fine resolutions (typically, minimum wall thickness of $\sim 140 \mu \mathrm{m}$ and minimum hole diameter of $\sim 200 \mu \mathrm{m})$. Moreover, PBF has especially shown amenability toward further miniaturization of printed parts, with feature sizes as low as $15-30 \mu \mathrm{m}$ and ARs as high as 260 reported recently ${ }^{53}$ using the "micro laser sintering" process.

As the processing workflow (Figure $1 \mathrm{a}-\mathrm{d}$ ) starts with a 3D printed metallic mold, it enjoys access to a greater design space than is possible with other methods of PDMS fabrication. For instance, if a thin, helical, wire-like structure is printed inside the cavity of the cylindrical metallic mold similar to the one shown in Figure 1a, the acidic etchant will attack the helical wire along with the walls of the mold, thus essentially "boring" a helical microchannel inside the resultant HAR PDMS cylinder. It is noteworthy that this additional structural complexity of a $3 \mathrm{D}$ microchannel can be created in the HAR pillar without any change in the processing workflow unlike other methods that are strictly restricted to a particular kind of microstructure, for example, soft lithography for planar microfluidic channels ${ }^{13}$ or deep drawing ${ }^{41,42}$ for HAR micropillars. We demonstrated the versatility of our workflow (Figure $1 \mathrm{a}-\mathrm{d}$ ) by fabricating different kinds of complex PDMS parts shown in Figures 2-4 that feature a broad range of microstructures ranging from complex biomimetic microstructures inspired by biological cilia sensors in nature (Figure 2); intricate 3D microfluidic channels inside HAR pillars (Figure 3); and finally, MEMS force, tactile, and flow sensors (Figure 4).

Fabrication of Complex PDMS Microstructures. Biomimetic HAR PDMS Micropillars. In nature, there exist numerous examples of HAR cilia structures made of natural soft polymer materials that demonstrate exceptional sensing performance and serve a variety of purposes such as flow ${ }^{35}$ and tactile $^{47}$ sensing. For instance, the slender morphology of arthropod filiform hair and fish neuromasts (Figure 2a) makes them sensitive to tiny flow disturbances on the order of $1 \mathrm{~mm} / \mathrm{s}$ in air $^{54}$ and $10 \mu \mathrm{m} / \mathrm{s}$ in water, ${ }^{55}$ respectively. Replicating these HAR structures using a soft polymeric material is thus essential for a true biomimetic structure. To this end, we used our methodology to successfully fabricate a slender PDMS micropillar (Figure 2a) with dimensions $(\mathrm{AR}=50$, diameter $=300$ $\mu \mathrm{m}$ ) that are usually outside the reach of typical PDMS fabrication techniques such as soft lithography, direct 3D printing, or existing sacrificial molding approaches. Further, the process can be easily extended to fabricate a $2 \mathrm{D}$ array of HAR PDMS micropillars that are useful, for instance, in biomimetic dry adhesives ${ }^{33}$ and directional flow sensors inspired by the stereocilia bundle ${ }^{35}$ occurring in mammalian (e.g., humans) and nonmammalian (e.g., bullfrog) vertebrates. Figure $2 \mathrm{~b}$ shows a scanning electron microscope image of a biological stereocilia in the bullfrog that consists of a bundle of 60 pillars of graded heights varying from 4 to $8 \mu \mathrm{m}$. ${ }^{35}$

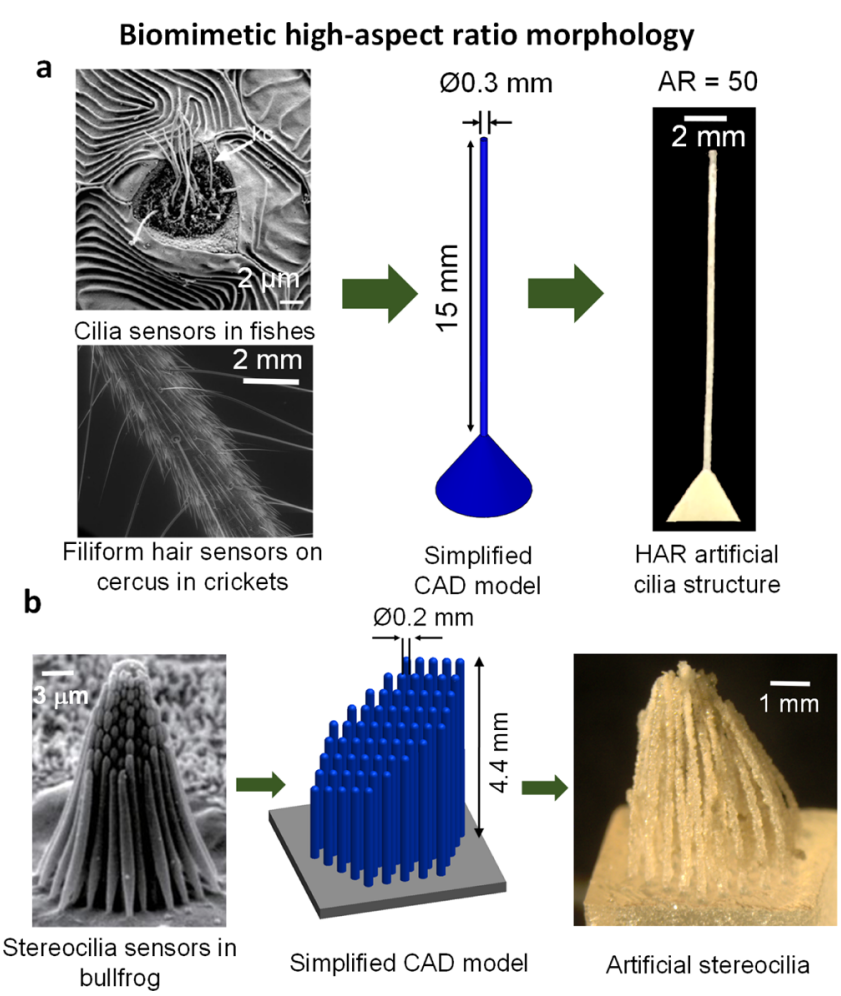

Figure 2. Biomimetic HAR structures and cilia sensors. (a) SEM images showing the HAR mechanosensory structures in cricket hair (left, bottom) and fish neuromasts (left, top). HAR biomimetic PDMS cilia structure $(A R=50)$ modeled in a CAD software (middle) and fabricated using the proposed method (right). (b) SEM image showing the intricate structural morphology of the saccular stereociliary hair bundles in a bullfrog (left). Array of micropillars mimicking the stereocilia structure (scaled up by $500 \times$ ) modeled in a CAD software (middle) and fabricated using the proposed method in PDMS (right). Image a (left, bottom) reproduced from Jacobs et al. ${ }^{56}$ with permission. Copyright 2008, The Company of Biologists. Image a (left, top) reproduced from $\mathrm{Webb}^{57}$ with permission. Copyright 2019, Taylor and Francis Group LLC. Image b (left) reproduced from Holt and Corey. ${ }^{58}$ Copyright 2000, National Academy of Sciences.

The biological structure was scaled up by a factor of 500 and modeled in CAD (Figure 2b, middle), with the tallest pillar being $200 \mu \mathrm{m}$ in diameter and $4.4 \mathrm{~mm}$ in height $(\mathrm{AR}=22)$, which was subsequently fabricated using our method (Figure $2 b$, right). The micropillars in Figure $2 b$ exhibited a "beady" appearance due to the imprint of the rough inner walls of the metallic mold, which is a common feature of the powder-based $3 \mathrm{D}$ printing process $(\mathrm{LPBF})$ used in this study. The ability to mimic biological structures such as the stereocilia offers an opportunity to not only build biomimetic sensors but to also form physical models that can help understand and explain biological phenomena such as the response of the bundle to a flow stimulus.

3D Microfluidic Channels. One of the major advantages of using $3 \mathrm{D}$ printing over soft lithography is its ability to create microfluidic channels in 3D space with little or no restrictions on the channel cross section or architecture. ${ }^{59} \mathrm{We}$ leveraged this by fabricating a single helical microchannel $(300 \mu \mathrm{m}$ channel diameter) and DNA-inspired double helical microchannels (250 $\mu \mathrm{m}$ channel diameter) running along the length of (and coaxial with) a PDMS cylinder (Figure 3a). The channels were injected with red (left) and red/blue (right) inks to enhance their visibility. The small size and flexibility of the PDMS structure is 
Microfluidic sensors and structures

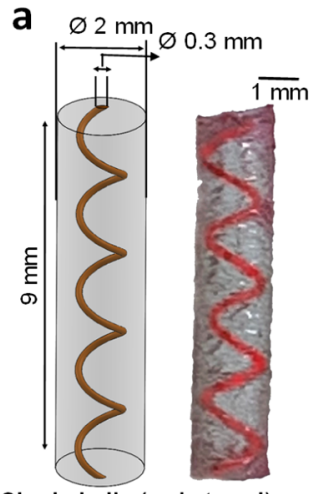

Single helix (red strand)

b

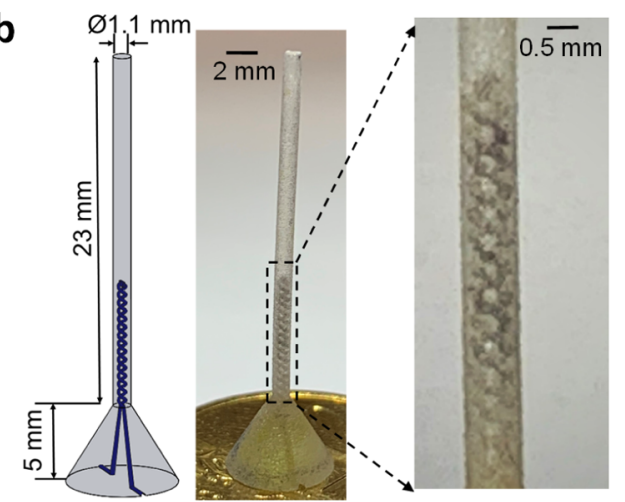

3D double helical microfluidic channels inside a HAR pillar

Figure 3. 3D microfluidic channels inside PDMS pillars. (a) Single helical $(300 \mu \mathrm{m}$ diameter $)$ and double helical $(250 \mu \mathrm{m}$ diameter $)$ microfluidic channels inside flexible PDMS pillars infused with red and red/blue inks, respectively, showing the size and flexibility of the structure (right). (b) A HAR PDMS pillar $(\mathrm{AR}=20)$ with a $3 \mathrm{D}$ microfluidic channel (channel diameter $=200 \mu \mathrm{m}$, total channel length $=27 \mathrm{~mm}$ ). The channel architecture comprised two coaxial double helices connected at the top with both the inlet and the outlet at the bottom.

also illustrated in Figure 3a (right). Further, we demonstrated the ability to incorporate two levels of structural complexities in a single structure: a single microchannel $(200 \mu \mathrm{m}$ channel diameter, $27 \mathrm{~mm}$ total double helix length) inside a HAR pillar (23 mm length, $1.1 \mathrm{~mm}$ pillar diameter) as shown in Figure $3 \mathrm{~b}$. Here, the microchannel (featuring two coaxial helices connected at the top) had both its inlet and outlet at opposite ends of the bottom fixture (Figure $3 \mathrm{~b}$ ). The successful fabrication of a 3D microfluidic channel inside a HAR pillar demonstrated the unparalleled ability of our methodology to fabricate soft material structures with multiple degrees of complexity that is not achievable using current processing methods.

Flexible MEMS Sensors with Embedded 3D Graphene Piezoresistors. The ability to create microchannels inside a PDMS structure, with significant design freedom in 3D space with respect to both the microfluidic channel architecture and the encapsulating PDMS structure, opens new possibilities of fabricating flexible MEMS sensors with embedded sensing elements. This embedding of sensing elements can be realized by infusing the microfluidic channels with an electrically conductive liquid such as graphite paint, ${ }^{44}$ silver-based liquid metal paste, ${ }^{46}$ and gallium-based liquid metal (e.g., Galinstan). ${ }^{60,61}$ The resulting flexible microstructure generates a capacitive or piezoresistive response upon the application of mechanical stimulus, thus effectively creating a 3D piezoresistor embedded within the PDMS structure. We explored these possibilities by infusing diluted conductive graphene nanoplatelet (GNP) dispersion (average nanoplatelet thickness $\sim 7$ $\mathrm{nm}$ ) into the microchannels fabricated using our method as shown in Figure 4. GNP dispersion was chosen as the piezoresistive sensing material owing to its high gauge factor ${ }^{49}$ caused by the ability of the nanoflakes (Figure $4 b$, bottom right) to slide easily over one another upon the application of strain. ${ }^{62}$ Figure 4a,b shows two different designs of MEMS flow sensors that feature HAR PDMS structures (hair-like structure in Figure $4 \mathrm{a}$ and cantilever in Figure 4b) featuring an embedded, serpentine, graphene-infused microchannel (150 $\mu \mathrm{m}$ channel diameter in Figure 4a and $300 \mu \mathrm{m}$ channel diameter in Figure 4b). In the presence of external stimuli such as fluid flow or mechanical touch, the HAR PDMS structure bends and causes a resistance change in the conductive GNP that can be calibrated with respect to the applied stimulus. Figure $4 \mathrm{c}$ shows a flexible tactile sensor array consisting of 25 nodes formed by 10 GNP piezoresistive channels ( $1 \mathrm{~mm}$ diameter) embedded into two rectangular PDMS blocks (five channels per block), a popular design for artificial skins. ${ }^{63}$ Finally, Figure 4d shows a MEMS force sensor featuring a GNP-infused microchannel embedded in a PDMS dome (diameter $=4 \mathrm{~mm}$ ), where the force applied on top of the dome is transduced into a resistance change in the strained GNP microchannel. The microchannel $(300 \mu \mathrm{m}$ channel diameter) consisted of a double helical shape connected at the top by a horizontal rectangular $(300 \mu \mathrm{m} \times 500 \mu \mathrm{m})$ microchannel. Figure 4 demonstrates the wide variety of sensor shapes, sizes, and functionalities that can be achieved using our methodology. Moreover, the embedment of the GNP piezoresistor inside the PDMS structure results in a naturally waterproof design, eliminating the need for any postfabrication packaging. To validate the efficacy of our method in fabricating flexible MEMS sensors, we conducted tests on the hair-like flow/tactile sensor (Figure 4a) and the dome-shaped force sensor (Figure 4d).

Bioinspired HAR Tactile/Flow Sensor. The design of the HAR flow sensor (Figure 4a) was inspired by the slender structures found in fishes and arthropods (Figure 2a, left) that act as ultrasensitive flow sensors and tactile feelers, respectively, and heighten their vigilance of the surroundings. Our processing method allowed us to incorporate the piezoresistive GNP sensing elements throughout the length of the hair-like structure (as opposed to only at the base), thus further enhancing the sensitivity to local stimuli. It must be noted that in previous bioinspired flow sensors, ${ }^{64}$ the sensing membrane (gold strain gauge patterned on a liquid crystal polymer membrane bonded to a silicon substrate) and the cilia-inspired pillar (3D-printed) were fabricated separately and bonded together later; contrary to this, the bioinspired sensor presented here was fabricated in a monolithic workflow without the need for additional assembly or packaging steps. The hair-like sensor was tested by subjecting it to oscillatory stimuli using a vibrating dipole apparatus that has been previously reported elsewhere. ${ }^{64}$ The vibration amplitude and frequency of the oscillating dipole (a stainless steel sphere of $8 \mathrm{~mm}$ radius) could be varied independently. In the presence of an external oscillatory stimulus, the PDMS pillar bent and caused a strain-induced resistance change in the GNP piezoresistor (Figure 5a) that was measured using a Wheatstone bridge circuit at a sampling frequency of $1 \mathrm{kHz}$. In the first experiment, the piezoresistive response of the sensor was measured by exciting the pillar tip using the oscillating dipole 


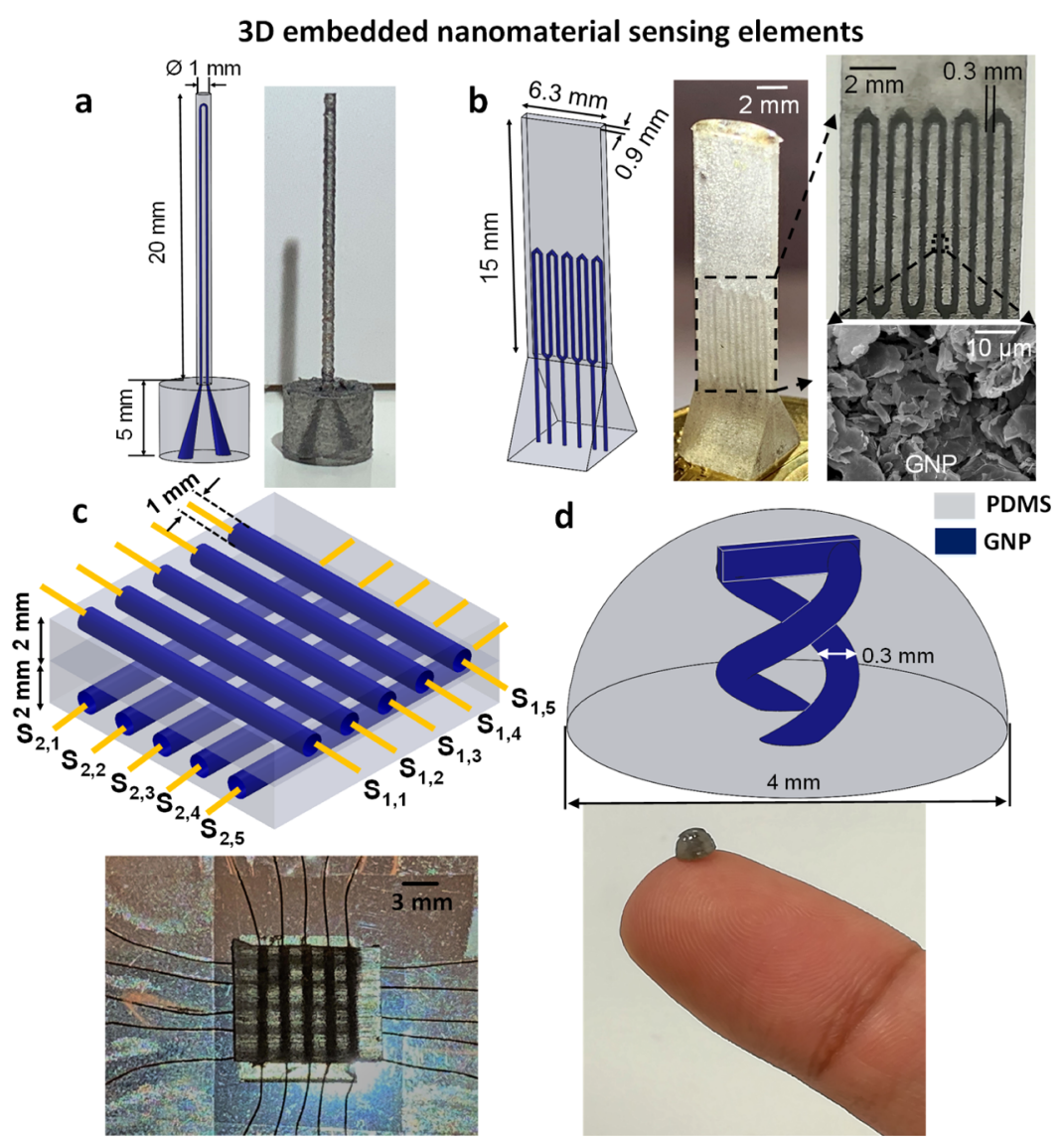

Figure 4. Flexible, waterproof, PDMS sensors with embedded GNP piezoresistors. (a) HAR PDMS hair-like structure featuring an embedded Ushaped GNP piezoresistor ( $150 \mu \mathrm{m}$ channel diameter) extending all the way along the length of the pillar. (b) HAR PDMS cantilever containing an embedded, serpentine microchannel (300 $\mu$ m channel diameter) before (middle) and after (right) GNP infusion, with the SEM image of the GNP shown in the inset. (c) Tactile sensor array featuring two PDMS blocks with five GNP-infused channels ( 1 mm diameter) each stacked on top of the other to form a popular design for artificial skins. ${ }^{63}$ (d) MEMS force sensor featuring a GNP-infused double helical microchannel (300 $\mu \mathrm{m}$ channel diameter).

(Figure 5a, inset) whose amplitude was varied (16 to $350 \mu \mathrm{m}$ ) at a frequency of $35 \mathrm{~Hz}$. The sensor output, defined as the Fast Fourier transform (FFT) peak at $35 \mathrm{~Hz}$ (assuming it existed) of the resulting time series data, was then plotted as a function of the excitation amplitude (Figure 5a). The sensor (base resistance $\sim 52 \mathrm{k} \Omega$ ) showed a linear response to varying tip displacements with a sensitivity of $\sim 1.5 \Omega / \mu \mathrm{m}$ and displayed an ability to sense oscillating amplitudes as low as $83 \mu \mathrm{m}$ at $35 \mathrm{~Hz}$.

Further, the flow sensing capability of the whisker sensor was tested by fixing the sensor horizontally $30 \mathrm{~mm}$ under water and exciting it at different flow velocities via the dipole that oscillated vertically above the sensor at a mean distance of $18 \mathrm{~mm}$ away from the whisker tip (Figure $5 b$, inset). Under water, the base resistance of the sensor changed to $\sim 70 \mathrm{k} \Omega$ (from $52 \mathrm{k} \Omega$ in air) due to buoyancy effects. An oscillating flow field was generated by varying the amplitude of the dipole at $35 \mathrm{~Hz}$ similar to the tactile experiment of Figure 5a. The root mean square (rms) flow velocity induced at the tip of the pillar by the oscillating dipole was estimated using the flow field model adapted from Goulet et al. $^{65}$

$$
v_{\mathrm{RMS}}=2.83 \pi f_{s}\left[\frac{d}{x}\right]^{3}
$$

where $f$ is the frequency of dipole oscillation $(35 \mathrm{~Hz}$ for this work), $s$ is the amplitude of dipole oscillation (calibrated using laser doppler vibrometry in past work ${ }^{64}$ for $f=35 \mathrm{~Hz}$ as shown in
Figure $\mathrm{S} 2), d$ is the sphere diameter (16 mm for this work), and $x$ is the distance of the hair tip from the oscillating dipole $(18 \mathrm{~mm}$ for this work). The flow sensor output displayed a parabolic relation with respect to the rms flow velocity consistent with the findings of Asadnia et al. ${ }^{64}$ who attributed this result to the dependence of the drag force on the square of the fluid velocity in the flow regime where the Reynolds number $(R e)$ exceeded 50. The $R e$ number in our experiments exceeded 50 at approximately $45 \mathrm{~mm} / \mathrm{s}$, which could explain the parabolic behavior of Figure $5 \mathrm{~b}$. The threshold detection limit was found to be as low as $13 \mathrm{~mm} / \mathrm{s}$ for the oscillatory water flow stimulus at $35 \mathrm{~Hz}$. Finally, a potential application of the sensor as a "drop counter" was explored (Figure 5c) where water drops of approximately $2 \mu \mathrm{L}$ volume were pipetted out onto the sensor tip from a height of $30 \mathrm{~mm}$ above the sensor every $30 \mathrm{~s}$. As seen from the results in Figure 5c, the sensor showed a clear resistance spike corresponding to each drop (average change in resistance $\sim 4.07 \pm 0.7 \mathrm{k} \Omega$ per drop) and good recovery over 10 evenly spaced drops, showing promise as a low-cost drop counter in, for instance, agricultural (e.g., irrigation) or biomedical (e.g., drip chamber) applications. The small variation in the resistance peaks may be attributed to the variability of water volume from drop to drop.

MEMS Force Sensor with a Double Helical GNP Piezoresistor. The MEMS force sensor shown in Figure $4 \mathrm{~d}$ was tested against a controlled load applied using a universal 

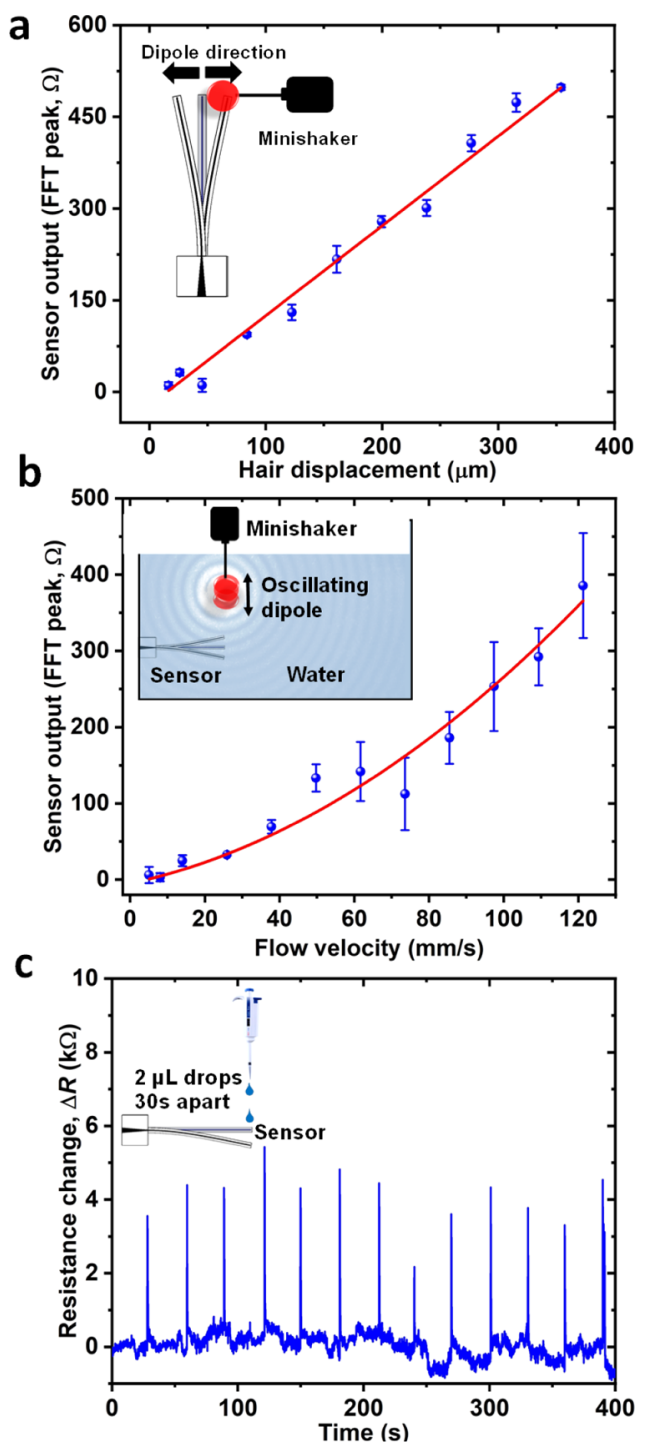

Figure 5. Experimental flow/tactile characterization of the bioinspired hair-like flow sensor. (a) Tactile sensing performance of the HAR whisker sensor. Linear sensor response (peak of FFT at $35 \mathrm{~Hz}$ ) measured as a function of the oscillatory tactile tip displacement of the sensor. (b) Oscillatory flow sensing in water. Quadratic sensor response (peak of FFT at $35 \mathrm{~Hz}$ ) measured as a function of the rms flow velocity at the tip of the hair-like whisker. (c) Possible application of the sensor as a "drop counter" registering a clear resistance spike of $\sim 4.07 \pm 0.7 \mathrm{k} \Omega$ per water drop.

microtribometer (UMT-3, Bruker, USA). The double helical shape of the 3D strain gauge allowed us to maximize the length of the piezoresistor within the small ( $4 \mathrm{~mm}$ diameter) PDMS dome for enhanced sensitivity. The sensor was subjected to a compressive force $(0-1.8 \mathrm{~N}$ in steps of $0.2 \mathrm{~N})$ applied for $30 \mathrm{~s}$ through a cylindrical pin (10 $\mathrm{mm}$ diameter): the load was linearly increased to its maximum value in $5 \mathrm{~s}$ and then maintained at this value for $25 \mathrm{~s}$ before it was released. As shown in Figure 6a, the application of the normal load strained the PDMS dome and the embedded GNP piezoresistor, thus inducing a change in resistance that was monitored using a Wheatstone bridge circuit at a sampling frequency of $10 \mathrm{~Hz}$. The sensor exhibited linear behavior up to a normal load of $1 \mathrm{~N}$ after which its output saturated (Figure 6b) and displayed a resolution of at least $0.2 \mathrm{~N}$ (Figure $6 \mathrm{c}$ ). The sensitivity (defined as the rate of change of fractional resistance with respect to the applied normal load) of our MEMS sensor in the 0-1 N range was measured to be $45 \% / \mathrm{N}$, which compared favorably with elastomeric piezoresistive force sensors reported in the literature, for example, $6.67 \% / \mathrm{N}$ by Pyo et al. ${ }^{66}$ and $18 \% / \mathrm{N}$ by Yin, ${ }^{67}$ whose sensor materials, dimensions, and normal load testing range were similar to those in this work.

Finally, we integrated the MEMS force sensor into the Philips 9000 series electric shaver to illustrate its application in realworld consumer goods. Skin irritation arising from shaving is among the most common cosmetic complaints of males in the USA and Europe. ${ }^{68}$ Shaving discomfort correlates directly with forces (usually around $2 \mathrm{~N}$ on average ${ }^{50}$ ) occurring during shaving, ${ }^{68}$ making it desirable to measure and quantify these forces. Electric shavers such as the Philips 9000 series used in this study are ideally required to operate with zero skin-cutter distance (SCD) to offer a close shave while minimizing skin irritation; however, direct measurement of the SCD is difficult and expensive, making it desirable to measure shaving forces from which the SCD can be inferred (and controlled, if needed) in real time. The MEMS sensor was integrated into the middle of the three rotary cutters of the electric shaver (Figure 7a) so that the sensor directly pressed against the skin during shaving and measured the normal force. The dome-shaped MEMS sensor was ideally suited for this application as it satisfied the requirements of size ( $4 \mathrm{~mm}$ dome small enough to fit in the center of the rotary cutters), flexibility (elastomeric PDMS), biocompatibility (PDMS structure), and force detection range (0-2 $\mathrm{N}$ from Figure $6 \mathrm{~b})$. Additional details regarding the system-level integration of the sensor into the shaver head and the wiring are presented in the Supporting Information (Figure S5). Tests were conducted where the user touched his face with the shaver switched on with a low, medium, and high intensity of pressure (Figure $7 \mathrm{~b}$ ). In the first test, the user was asked to hold the shaver to his face for a period of $5 \mathrm{~s}$ and then release the shaver (Figure 7c), while in the second test, the user was asked to touch the shaver to his face and release it immediately without holding (Figure 7d). A video of the press-hold-release tests can be accessed in the Supporting Information (Video S1). As seen in Figure $7 c, d$, the sensor was able to differentiate between different shaving pressures exerted by the user. The data can be used in the future in a variety of ways, for example, (i) to warn the user if too much (or too little) shaving pressure is being applied, (ii) to take corrective action through a feedback loop to ensure that the SCD remains close to zero, and (iii) to customize the shaving experience by adjusting the "ideal" shaving pressure based on the individual user's skin type.

\section{CONCLUSIONS AND OUTLOOK}

We developed a novel processing technique (entailing metal 3D printing of a thin-walled mold, soft polymer casting, and acidic etching) to fabricate a variety of complex PDMS structures. We demonstrated the facile fabrication of microfluidic channels inside HAR structures and further infused them with high-gauge factor GNPs to realize piezoresistive MEMS sensors with embedded sensing elements. Two sensors, namely, a HAR "hairlike" flow/tactile sensor and a dome-like force sensor, were tested against controlled stimuli and displayed excellent performance. Finally, the utility of the dome-like MEMS force sensor was demonstrated by integrating it into a personal electric shaver. The sensor successfully detected varying degrees of pressure applied by the shaver on the user's skin and thus 
a

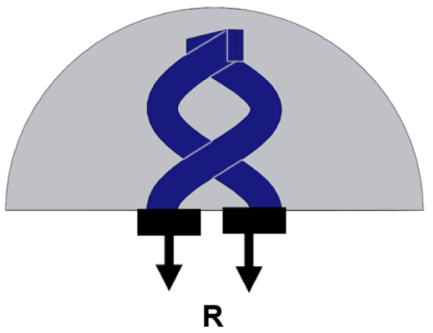

b

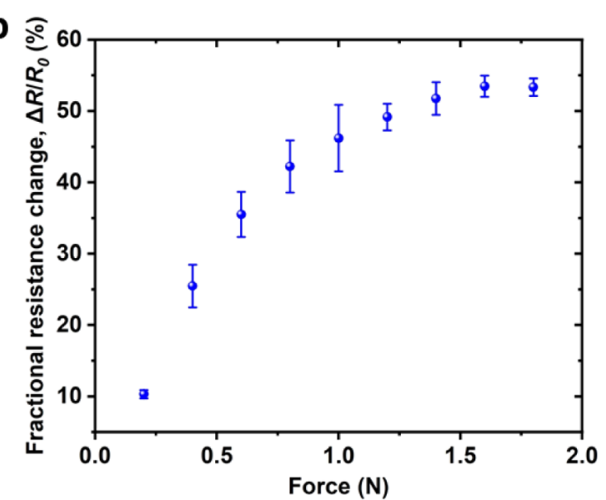

Piston $(F=0 \sim 2 N)$

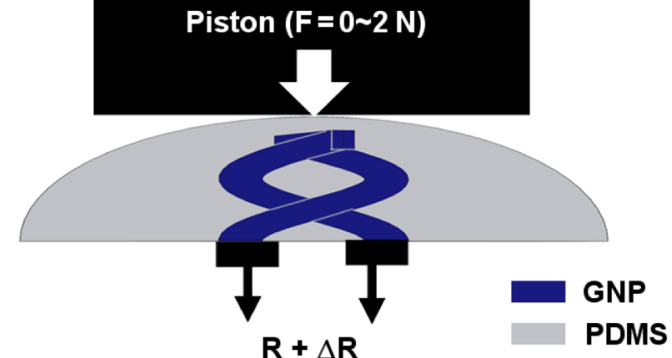

c

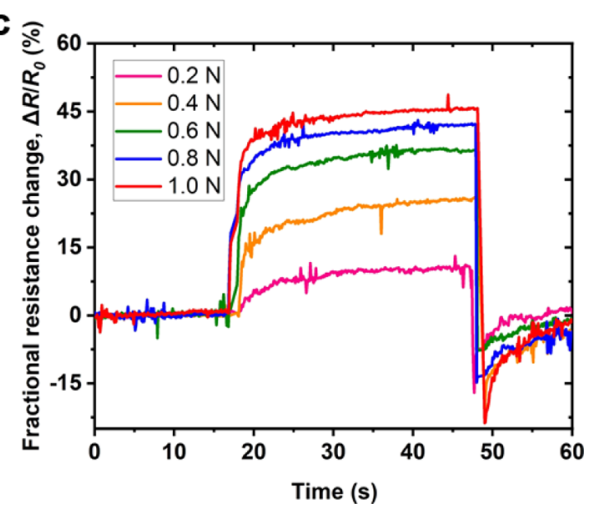

Figure 6. Dome-shaped MEMS force sensor with double helical GNP piezoresistor. (a) Mechanism of piezoresistive force sensing (the deformation of the dome is exaggerated in the schematic). (b) Sensor output as a function of load applied on the dome. (c) Time series data of sensor resistance change for five representative loads for a typical $30 \mathrm{~s}$ test ( $5 \mathrm{~s}$ to ramp up to maximum load, $25 \mathrm{~s}$ hold at the load, and then release).
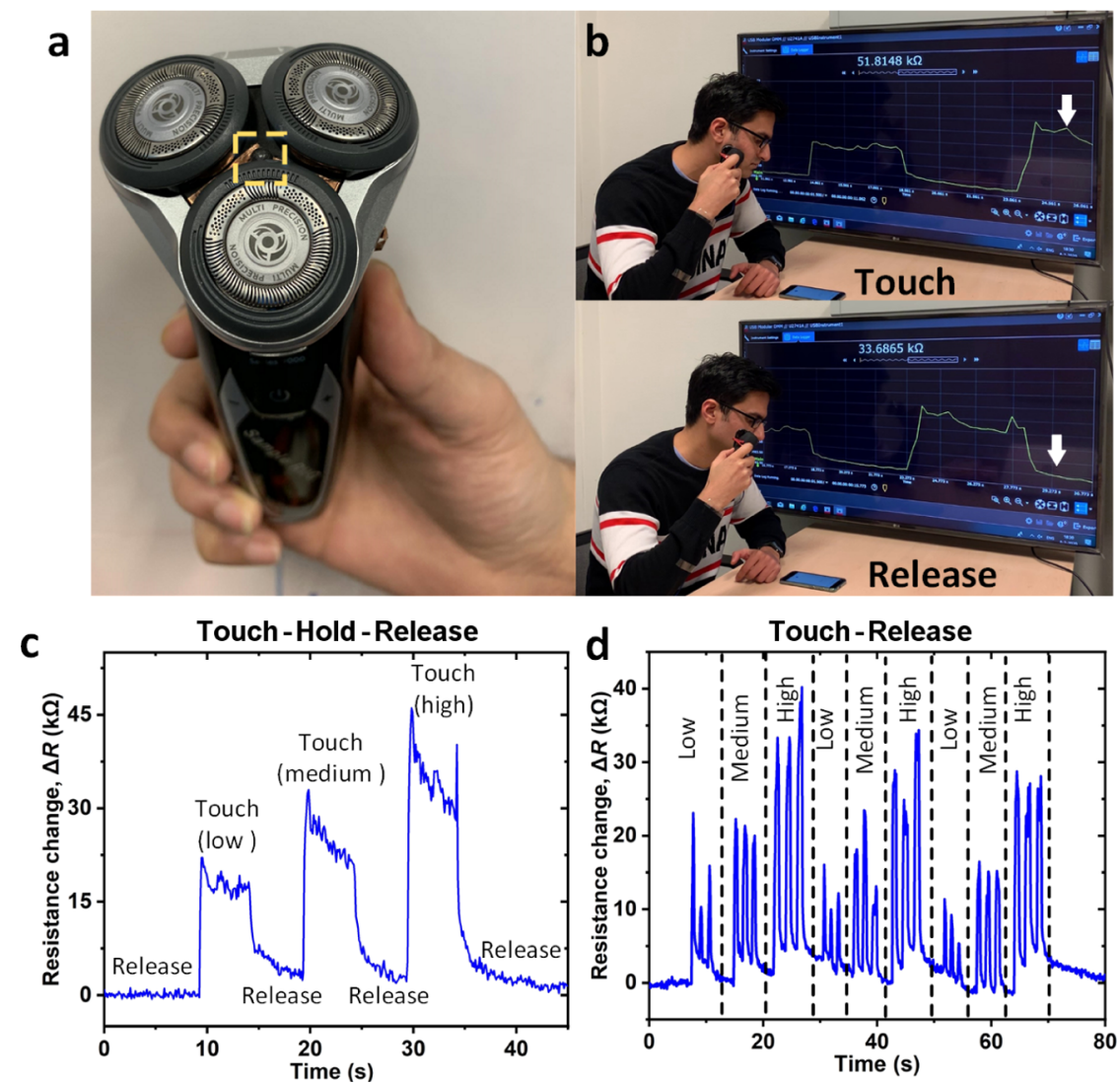

Figure 7. Measurement of normal loads during shaving using the MEMS force sensor. (a) Integration of the MEMS sensor (dotted yellow box) between the three rotary blades of the Philips 9000 series electric shaver. (b) The user pressing the shaver onto his face and releasing it while the data acquisition system on the screen shows the sensor output in real time (current resistance value shown by white arrow). (c) Sensor output when the shaver was pressed against the face for $5 \mathrm{~s}$ with varying degrees of intensity (static test). (d) Sensor output when the shaver was touched and released immediately with varying degrees of intensity (dynamic test). 
showed promise for integration into "smart" consumer products in the future.

Our processing approach was thus found to be versatile in fabricating a variety of PDMS structures. The use of metal instead of previously used materials (ABS, PVA, wax, sugar, etc.) for the sacrificial mold allowed us to achieve fine feature sizes $(\sim 150 \mu \mathrm{m})$ and very HARs (at least 50$)$ in a variety of bioinspired PDMS structures and 3D microfluidic channel architectures. Although we used PDMS in this study, the process can be easily extended to other soft polymers that are difficult to 3D print directly. While most PDMS fabrication techniques are developed for and suited to a specific kind of structure, for example, microfluidic channels, membranes, HAR pillars, and so on, our method is general enough to fabricate any design (subject to the limitations of the metal 3D printing process) using the same processing workflow, thus vastly expanding the design space for soft material fabrication. The use of this method is especially suited for fabricating biomimetic flow sensors that typically feature HAR structures (e.g., fish neuromasts, arthropod filiform hairs) and intricate geometric shapes (e.g., seal whiskers). Moreover, the ability to create $3 \mathrm{D}$ microchannels followed by the infusion of a conducting liquid offers a naturally waterproof sensor structure with no need for additional assembly or packaging steps. The recent trends toward industrialization (enabling process automation and batch fabrication compatibility) and miniaturization (allowing minimum feature sizes of $15-30 \mu \mathrm{m}$ ) in metal 3D printing can thus enable our processing workflow to be used as a scalable and cleanroom-free method for the low-cost fabrication of 3D soft polymeric structures and flexible MEMS sensors.

\section{MATERIALS AND METHODS}

Metal 3D Printing. The CAD model of the thin-walled mold was created as the "negative" of the desired PDMS structure and was printed in the SLM $125^{\mathrm{HL}}$ (SLM Solutions AG, Germany) machine using 17-4 PH stainless steel powder (LPW Technology, UK, $10-45 \mu \mathrm{m}$ size distribution) as the raw material. Manufacturer-recommended processing parameters were used in all the experiments. After 3D printing, the mold was ultrasonically cleaned using compressed air and ultrasonic cleaning in acetone to remove any remnant powder particles inside the mold cavity.

PDMS Drop-Casting and Curing. Commercially obtained PDMS solution (Sylgard 184) was prepared in the ratio of 10 parts base monomer to 1 part curing agent by weight, mixed thoroughly, and degassed in a vacuum chamber for $15 \mathrm{~min}$. The PDMS solution was then drawn up in a syringe and drop-cast into the metallic mold using a 20-27 G needle (depending upon the dimension of the mold cavity). The mold filled with PDMS was degassed under vacuum again for $5 \mathrm{~min}$ and then heated inside a furnace at $120{ }^{\circ} \mathrm{C}$ for $20 \mathrm{~min}$ to cure the PDMS. The outer walls of the mold were lightly ground with sandpaper to remove traces of cured PDMS adhered to it, if any.

Graphene Infusion into Sensor Microchannels. Commercially obtained conductive graphene dispersion (Graphene Laboratories Inc., USA) containing GNP (average thickness $\sim 7 \mathrm{~nm}, 23 \mathrm{wt} \%$ graphene in $n$-butyl acetate solvent) was diluted with ethanol ( 1 part graphene dispersion, 4 parts ethanol by volume). The diluted GNP solution was then injected into the microchannels of both sensors using a $22 \mathrm{G}$ needle. The microchannels in both sensors were made wider at the base $(\sim 1 \mathrm{~mm})$ to ease the infusion process and avoid cleavage of the PDMS due to the needle. The structure was then gently annealed at $100{ }^{\circ} \mathrm{C}$ for $1 \mathrm{~h}$ to improve the conductivity of the GNP and form an electrically conductive path inside the PDMS structure.

Oscillating Dipole Apparatus. The oscillatory stimuli for the tactile and flow tests were generated using a permanent magnet minishaker (model 4810, Brüel \& Kjær, Denmark) setup. The apparatus comprised a function generator, a power amplifier, and an oscillating membrane to which the stainless steel sphere ( $8 \mathrm{~mm}$ radius) was attached via a stainless steel rod. The excitation voltage to the function generator could be controlled and amplified by the user. The calibration of the oscillating dipole setup using laser doppler vibrometry (reported in previous work ${ }^{64}$ ) allowed us to vary the amplitude of oscillation precisely at a frequency of $35 \mathrm{~Hz}$ and a voltage gain of $20 \mathrm{~dB}$. The calibration curve is shown in Figure S4 of the Supporting Information.

Sensor Testing and Data Acquisition. The sensors were mounted on one end of a microscopic glass slide and the free ends of the microchannels at the sensor base were connected to copper leads (conductive copper tapes) via conductive silver paste (EPO-TEK H20E, Epoxy Technology Inc., USA). Finally, the circumference of the sensor base was sealed with PDMS to secure the sensors to the glass slide and make them waterproof. The two copper leads from the sensors were connected to a Wheatstone bridge circuit powered by a $7 \mathrm{~V}$ battery and the voltage output from the bridge was read using a data acquisition system (NI-DAQ UBS 6003, National Instruments, USA) and the NI Signal Express software. For the dynamic tactile/flow tests (Figure $5 a, b)$, each experiment at a particular dipole vibration amplitude was repeated three times for at least 350 excitation cycles. The sensor output at the excitation frequency $(35 \mathrm{~Hz})$ was calculated by converting the raw time series data ( $1 \mathrm{kHz}$ sampling frequency) to the frequency domain using the FFT operation (Origin 2020, OriginLab, USA) and noting the height of the peak at that excitation frequency in the frequency domain, assuming the peak existed. For the static tests conducted on the MEMS force sensor (Figure 6), data were recorded at a frequency of $10 \mathrm{~Hz}$. Each experiment at a particular load was repeated thrice and the average fractional resistance change was plotted in Figure $6 \mathrm{c}$ as a function of the load. In all the sensor tests, the raw voltage data from the Wheatstone bridge circuit was converted to instantaneous sensor resistance using Kirchhoff's laws.

\section{ASSOCIATED CONTENT}

\section{Supporting Information}

The Supporting Information is available free of charge at https://pubs.acs.org/doi/10.1021/acsami.0c21295.

Effect of acidic treatment on the swelling, surface texture, and mechanical properties of PDMS; fabrication of multimaterial structures using the proposed methodology; calibration curve of the minishaker dipole setup; the integration of the MEMS force sensor into the Philips 9000 series shaver head; and a video demonstration of the sensorized electric shaver during operation (PDF)

Real-time demonstration of a sensorized shaver at different load cycles (MP4)

\section{AUTHOR INFORMATION}

\section{Corresponding Author}

Amar M. Kamat - Advanced Production Engineering, Engineering and Technology Institute Groningen, Faculty of Science and Engineering, University of Groningen, Groningen 9747 AG, The Netherlands; (1) orcid.org/0000-0002-16229067; Phone: +31 641490186; Email: a.m.kamat@rug.nl

\section{Authors}

Yutao Pei - Advanced Production Engineering, Engineering and Technology Institute Groningen, Faculty of Science and Engineering, University of Groningen, Groningen 9747 AG, The Netherlands; 이이이.org/0000-0002-1817-2228

Bayu Jayawardhana - Discrete Technology and Production Automation, Engineering and Technology Institute Groningen, Faculty of Science and Engineering, University of Groningen, Groningen 9747 AG, The Netherlands

Ajay Giri Prakash Kottapalli - Advanced Production Engineering, Engineering and Technology Institute Groningen, 
Faculty of Science and Engineering, University of Groningen, Groningen 9747 AG, The Netherlands; MIT Sea Grant College Program, Massachusetts Institute of Technology (MIT), Cambridge 02139, Massachusetts, United States; (1) orcid.org/0000-0002-3868-7069

Complete contact information is available at:

https://pubs.acs.org/10.1021/acsami.0c21295

\section{Author Contributions}

A.M.K. and A.G.P.K. conceived the idea and designed the experimental plan. A.M.K. performed the fabrication, data analysis, and wrote the manuscript. A.M.K. and A.G.P.K performed the sensor testing. A.G.P.K prepared the figures for the manuscript. Y.P., B.J., and A.G.P.K. supervised the project and edited the manuscript. All the authors contributed to the discussion of results and a critical reading of the manuscript.

Notes

The authors declare no competing financial interest.

\section{ACKNOWLEDGMENTS}

This research was partially carried out under project number S16044 in the framework of the Partnership Program of the Materials innovation institute M2i (www.m2i.nl) and the Technology Foundation STW (www.stw.nl), which is part of the Netherlands Organization for Scientific Research (www. nwo.nl). Part of this work was labeled by ITEA and funded by local authorities under the grant agreement ITEA-2018-17030Daytime. A.G.P.K. acknowledges the startup grant awarded by the University of Groningen. The authors gratefully thank Dr. Prashant Sharma (UMCG, Groningen) for granting access to his experimental facilities for pressure sensor testing and acknowledge valuable discussions with Martijn van Zutphen (Philips Consumer Lifestyle, Drachten), Dr. Wilbert van den Eijnde (NHL Stenden Hogeschool, Emmen), and Eelco Galestien (Philips Consumer Lifestyle, Drachten).

\section{REFERENCES}

(1) ASTM F2792-Standard Terminology for Additive Manufacturing Technologies; ASTM International; 2013.

(2) Kelly, B. E.; Bhattacharya, I.; Heidari, H.; Shusteff, M.; Spadaccini, C. M.; Taylor, H. K. Volumetric Additive Manufacturing via Tomographic Reconstruction. Science 2019, 363, 1075-1079.

(3) Debroy, T.; Wei, H. L.; Zuback, J. S.; Mukherjee, T.; Elmer, J. W.; Milewski, J. O.; Beese, A. M.; Wilson-heid, A.; De, A.; Zhang, W. Additive Manufacturing of Metallic Components-Process, Structure and Properties. Prog. Mater. Sci. 2018, 92, 112-224.

(4) Chen, Z.; Li, Z.; Li, J.; Liu, C.; Lao, C.; Fu, Y.; Liu, C.; Li, Y.; Wang, P.; He, Y. 3D Printing of Ceramics: A Review. J. Eur. Ceram. Soc. 2019, 39, 661-687.

(5) Ligon, S. C.; Liska, R.; Stampfl, J.; Gurr, M.; Mülhaupt, R. Polymers for 3D Printing and Customized Additive Manufacturing. Chem. Rev. 2017, 117, 10212-10290.

(6) Truby, R. L.; Lewis, J. A. Printing Soft Matter in Three Dimensions. Nature 2016, 540, 371-378.

(7) McDonald, J. C.; Whitesides, G. M. Poly(Dimethylsiloxane) as a Material for Fabricating Microfluidic Devices. Acc. Chem. Res. 2002, 35, 491-499.

(8) Huh, D.; Kim, H. J.; Fraser, J. P.; Shea, D. E.; Khan, M.; Bahinski, A.; Hamilton, G. A.; Ingber, D. E. Microfabrication of Human Organson-Chips. Nat. Protoc. 2013, 8, 2135-2157.

(9) De Jong, J.; Lammertink, R. G. H.; Wessling, M. Membranes and Microfluidics: A Review. Lab Chip 2006, 6, 1125-1139.

(10) Palchesko, R. N.; Zhang, L.; Sun, Y.; Feinberg, A. W. Development of Polydimethylsiloxane Substrates with Tunable Elastic
Modulus to Study Cell Mechanobiology in Muscle and Nerve. PLoS One 2012, 7, No. e51499.

(11) Shepherd, R. F.; Ilievski, F.; Choi, W.; Morin, S. A.; Stokes, A. A.; Mazzeo, A. D.; Chen, X.; Wang, M.; Whitesides, G. M. Multigait Soft Robot. Proc. Natl. Acad. Sci. U.S.A. 2011, 108, 20400-20403.

(12) Kumar, K. S.; Chen, P.-Y.; Ren, H. A Review of Printable Flexible and Stretchable Tactile Sensors. Research 2019, 2019, 3018568.

(13) Xia, Y.; Whitesides, G. M. Soft Lithography. Angew. Chem., Int. Ed. 1998, 37, 550-575.

(14) Au, A. K.; Huynh, W.; Horowitz, L. F.; Folch, A. 3D-Printed Microfluidics. Angew. Chem., Int. Ed. 2016, 55, 3862-3881.

(15) Chen, C.; Mehl, B. T.; Munshi, A. S.; Townsend, A. D.; Spence, D. M.; Martin, R. S. 3D-Printed Microfluidic Devices: Fabrication, Advantages and Limitations-a Mini Review. Anal. Methods 2016, 8, $6005-6012$

(16) Song, S.-H.; Lee, C.-K.; Kim, T.-J.; Shin, I.-c.; Jun, S.-C.; Jung, H.I. A Rapid and Simple Fabrication Method for 3-Dimensional Circular Microfluidic Channel Using Metal Wire Removal Process. Microfluid. Nanofluid. 2010, 9, 533-540.

(17) Singh, R. K.; Kumar, A.; Kant, R.; Gupta, A.; Suresh, E.; Bhattacharya, S. Design and Fabrication of 3-Dimensional Helical Structures in Polydimethylsiloxane for Flow Control Applications. Microsyst. Technol. 2014, 20, 101-111.

(18) Hirama, H.; Odera, T.; Torii, T.; Moriguchi, H. A LithographyFree Procedure for Fabricating Three-Dimensional Microchannels Using Hydrogel Molds. Biomed. Microdevices 2012, 14, 689-697.

(19) Liu, X.; Wang, Q.; Qin, J.; Lin, B. A Facile "Liquid-Molding" Method to Fabricate PDMS Microdevices with 3-Dimensional Channel Topography. Lab Chip 2009, 9, 1200-1205.

(20) He, Y.; Qiu, J.; Fu, J.; Zhang, J.; Ren, Y.; Liu, A. Printing 3D Microfluidic Chips with a 3D Sugar Printer. Microfluid. Nanofluid. 2015, 19, 447-456.

(21) Gelber, M. K.; Bhargava, R. Monolithic Multilayer Microfluidics via Sacrificial Molding of 3D-Printed Isomalt. Lab Chip 2015, 15, $1736-1741$

(22) Saggiomo, V.; Velders, A. H. Simple 3D Printed ScaffoldRemoval Method for the Fabrication of Intricate Microfluidic Devices. Adv. Sci. 2015, 2, 1500125.

(23) Li, Z.; Yang, J.; Li, K.; Zhu, L.; Tang, W. Fabrication of PDMS Microfluidic Devices with 3D Wax Jetting. RSC Adv. 2017, 7, 33133320.

(24) Hwang, Y.; Paydar, O. H.; Candler, R. N. 3D Printed Molds for Non-Planar PDMS Microfluidic Channels. Sens. Actuators, A 2015, 226, $137-142$.

(25) Dahlberg, T.; Stangner, T.; Zhang, H.; Wiklund, K.; Lundberg, P.; Edman, L.; Andersson, M. 3D Printed Water-Soluble Scaffolds for Rapid Production of PDMS Micro-Fluidic Flow Chambers. Sci. Rep. 2018, 8, 1-10.

(26) Goh, W. H.; Hashimoto, M. Fabrication of 3D Microfluidic Channels and In-Channel Features Using 3D Printed, Water-Soluble Sacrificial Mold. Macromol. Mater. Eng. 2018, 303, 1700484.

(27) Femmer, T.; Kuehne, A. J. C.; Torres-Rendon, J.; Walther, A.; Wessling, M. Print Your Membrane: Rapid Prototyping of Complex 3D-PDMS Membranes via a Sacrificial Resist. J. Membr. Sci. 2015, 478, $12-18$.

(28) Kolesky, D. B.; Truby, R. L.; Gladman, A. S.; Busbee, T. A.; Homan, K. A.; Lewis, J. A. 3D Bioprinting of Vascularized, Heterogeneous Cell-Laden Tissue Constructs. Adv. Mater. 2014, 26, 3124-3130.

(29) Roh, S.; Parekh, D. P.; Bharti, B.; Stoyanov, S. D.; Velev, O. D. 3D Printing by Multiphase Silicone/Water Capillary Inks. Adv. Mater. 2017, 29, 1701554.

(30) Femmer, T.; Kuehne, A. J. C.; Wessling, M. Print Your Own Membrane: Direct Rapid Prototyping of Polydimethylsiloxane. Lab Chip 2014, 14, 2610-2613.

(31) Hinton, T. J.; Hudson, A.; Pusch, K.; Lee, A.; Feinberg, A. W. 3D Printing PDMS Elastomer in a Hydrophilic Support Bath via Freeform Reversible Embedding. ACS Biomater. Sci. Eng. 2016, 2, 1781-1786. 
(32) Ozbolat, V.; Dey, M.; Ayan, B.; Povilianskas, A.; Demirel, M. C.; Ozbolat, I. T. 3D Printing of PDMS Improves Its Mechanical and Cell Adhesion Properties. ACS Biomater. Sci. Eng. 2018, 4, 682-693.

(33) Li, Y.; Krahn, J.; Menon, C. Bioinspired Dry Adhesive Materials and Their Application in Robotics: A Review. J. Bionic Eng. 2016, 13, 181-199.

(34) Toonder, J. M. J. d.; Onck, P. R. Microfluidic Manipulation with Artificial/Bioinspired Cilia. Trends Biotechnol. 2013, 31, 85-91.

(35) Asadnia, M.; Kottapalli, A. G. P.; Karavitaki, K. D.; Warkiani, M. E.; Miao, J.; Corey, D. P.; Triantafyllou, M. From Biological Cilia to Artificial Flow Sensors: Biomimetic Soft Polymer Nanosensors with High Sensing Performance. Sci. Rep. 2016, 6, 1-13.

(36) Peleshanko, S.; Julian, M. D.; Ornatska, M.; McConney, M. E.; LeMieux, M. C.; Chen, N.; Tucker, C.; Yang, Y.; Liu, C.; Humphrey, J. A. C.; Tsukruk, V. V. Hydrogel-Encapsulated Microfabricated Haircells Mimicking Fish Cupula Neuromast. Adv. Mater. 2007, 19, 2903-2909.

(37) McConney, M. E.; Chen, N.; Lu, D.; Hu, H. A.; Coombs, S.; Liu, C.; Tsukruk, V. V. Biologically Inspired Design of Hydrogel-Capped Hair Sensors for Enhanced Underwater Flow Detection. Soft Matter 2009, 5, 292-295.

(38) Große, S.; Schröder, W. The Micro-Pillar Shear-Stress Sensor MPS3 for Turbulent Flow. Sensors 2009, 9, 2222-2251.

(39) Sasoglu, F. M.; Bohl, A. J.; Layton, B. E. Design and Microfabrication of a High-Aspect-Ratio PDMS Microbeam Array for Parallel Nanonewton Force Measurement and Protein Printing. J. Micromech. Microeng. 2007, 17, 623-632.

(40) Waghule, T.; Singhvi, G.; Dubey, S. K.; Pandey, M. M.; Gupta, G.; Singh, M.; Dua, K. Microneedles: A Smart Approach and Increasing Potential for Transdermal Drug Delivery System. Biomed. Pharmacother. 2019, 109, 1249-1258.

(41) Paek, J.; Kim, J. Microsphere-Assisted Fabrication of High Aspect-Ratio Elastomeric Micropillars and Waveguides. Nat. Commun. 2014, 5, 1-8.

(42) Li, Q.; Dhakal, R.; Kim, J. Microdroplet-Based On-Demand Drawing of High Aspect-Ratio Elastomeric Micropillar and Its Contact Sensing Application. Sci. Rep. 2017, 7, 1-10.

(43) Shao, G.; Wu, J.; Cai, Z.; Wang, W. Fabrication of Elastomeric High-Aspect-Ratio Microstructures Using Polydimethylsiloxane (PDMS) Double Casting Technique. Sens. Actuators, A 2012, 178, 230-236.

(44) Dijkshoorn, A.; Werkman, P.; Welleweerd, M.; Wolterink, G.; Eijking, B.; Delamare, J.; Sanders, R.; Krijnen, G. J. M. Embedded Sensing: Integrating Sensors in 3-D Printed Structures. J. Sens. Sens. Syst. 2018, 7, 169-181.

(45) Muth, J. T.; Vogt, D. M.; Truby, R. L.; Mengüç, Y.; Kolesky, D. B.; Wood, R. J.; Lewis, J. A. Embedded 3D Printing of Strain Sensors within Highly Stretchable Elastomers. Adv. Mater. 2014, 26, 63076312.

(46) Wu, S.-Y.; Yang, C.; Hsu, W.; Lin, L. 3D-Printed Microelectronics for Integrated Circuitry and Passive Wireless Sensors. Microsyst. Nanoeng. 2015, 1, 1-9.

(47) Dechant, H. E.; Rammerstorfer, F. G.; Barth, F. G. Arthropod Touch Reception: Stimulus Transformation and Finite Element Model of Spider Tactile Hairs. J. Comp. Physiol., A 2001, 187, 313-322.

(48) Mogdans, J.; Engelmann, J.; Hanke, W.; Kröther, S. The Fish Lateral Line: How to Detect Hydrodynamic Stimuli BT. Sensors and Sensing in Biology and Engineering; Barth, F. G., Humphrey, J. A. C., Secomb, T. W., Eds.; Springer Vienna: Vienna, 2003; pp 173-185.

(49) Kamat, A. M.; Pei, Y.; Kottapalli, A. G. P. Bioinspired Cilia Sensors with Graphene Sensing Elements Fabricated Using 3D Printing and Casting. Nanomaterials 2019, 9, 954.

(50) Cowley, K.; Vanoosthuyze, K. The Biomechanics of Blade Shaving. Int. J. Cosmet. Sci. 2016, 38, 17-23.

(51) Nageswara Rao, P.; Kunzru, D. Fabrication of Microchannels on Stainless Steel by Wet Chemical Etching. J. Micromech. Microeng. 2007, 17, N99-N106.

(52) Lee, J. N.; Park, C.; Whitesides, G. M. Solvent Compatibility of Poly(Dimethylsiloxane)-Based Microfluidic Devices. Anal. Chem. 2003, 75, 6544-6554.
(53) Nagarajan, B.; Hu, Z.; Song, X.; Zhai, W.; Wei, J. Development of Micro Selective Laser Melting: The State of the Art and Future Perspectives. Engineering 2019, 5, 702-720.

(54) Humphrey, J. A. C.; Barth, F. G.; Reed, M.; Spak, A. The Physics of Arthropod Medium-Flow Sensitive Hairs: Biological Models for Artificial Sensors BT. Sensors and Sensing in Biology and Engineering; Barth, F. G., Humphrey, J. A. C., Secomb, T. W., Eds.; Springer Vienna: Vienna, 2003; pp 129-144.

(55) Kroese, A. B. A.; Van der Zalm, J. M.; Van den Bercken, J. Frequency Response of the Lateral-Line Organ of Xenopus Laevis. Pflugers Arch. 1978, 375, 167-175.

(56) Jacobs, G. A.; Miller, J. P.; Aldworth, Z. Computational Mechanisms of Mechanosensory Processing in the Cricket. J. Exp. Biol. 2008, 211, 1819-1828.

(57) Webb, J. Morphology of the Mechanosensory Lateral Line System of Fishes. In The Senses: A Comprehensive Resource, 2nd ed.; Fritzsch, B., Ed.; Elsevier, 2020.

(58) Holt, J. R.; Corey, D. P. Two Mechanisms for Transducer Adaptation in Vertebrate Hair Cells. Proc. Natl. Acad. Sci. U.S.A. 2000, 97, 11730-11735.

(59) Bhattacharjee, N.; Urrios, A.; Kang, S.; Folch, A. The Upcoming 3D-Printing Revolution in Microfluidics. Lab Chip 2016, 16, 17201742.

(60) Wissman, J. P.; Sampath, K.; Freeman, S. E.; Rohde, C. A. Capacitive Bio-Inspired Flow Sensing Cupula. Sensors 2019, 19, 2639.

(61) Zhou, X.-P.; Liu, C.; Zhao, K. A Novel Liquid Metal Sensor with Three Microchannels Embedded in Elastomer. Smart Mater. Struct. 2020, 29, 045011.

(62) Hempel, M.; Nezich, D.; Kong, J.; Hofmann, M. A Novel Class of Strain Gauges Based on Layered Percolative Films of 2D Materials. Nano Lett. 2012, 12, 5714-5718.

(63) Lipomi, D. J.; Vosgueritchian, M.; Tee, B. C.-K.; Hellstrom, S. L.; Lee, J. A.; Fox, C. H.; Bao, Z. Skin-like Pressure and Strain Sensors Based on Transparent Elastic Films of Carbon Nanotubes. Nat. Nanotechnol. 2011, 6, 788-792.

(64) Asadnia, M.; Kottapalli, A. G. P.; Miao, J.; Warkiani, M. E.; Triantafyllou, M. S. Artificial Fish Skin of Self-Powered MicroElectromechanical Systems Hair Cells for Sensing Hydrodynamic Flow Phenomena. J. R. Soc., Interface 2015, 12, 20150322.

(65) Goulet, J.; Engelmann, J.; Chagnaud, B. P.; Franosch, J.-M. P.; Suttner, M. D.; Van Hemmen, J. L. Object Localization through the Lateral Line System of Fish: Theory and Experiment. J. Comp. Physiol., A 2008, 194, 1-17.

(66) Pyo, S.; Lee, J.-I.; Kim, M.-O.; Chung, T.; Oh, Y.; Lim, S.-C.; Park, J.; Kim, J. Development of a Flexible Three-Axis Tactile Sensor Based on Screen-Printed Carbon Nanotube-Polymer Composite. J. Micromech. Microeng. 2014, 24 (). DOI: 10.1088/0960-1317/24/7/ 075012.

(67) Yin, J. Soft Tactile Sensor Embedded Artificial Skin; University of Washington, 2017.

(68) Elsner, P. Overview and Trends in Male Grooming. Br. J. Dermatol. 2012, 166, 2-5. 